\title{
First-line ultrasound assessment of penetrating Crohn's disease in pediatric patients
}

Authors: Małgorzata Nowakowska ${ }^{1}$, Maryla Kuczyńska ${ }^{1}$, Monika Zbroja $^{1}$, Weronika Cyranka ${ }^{1}$, Monika Piekarska ${ }^{1}$, Magdalena Grzegorczyk ${ }^{1}$, Karolina Siejka ${ }^{1}$, Magdalena Woźniak ${ }^{2}$ (mentor), Agnieszka Brodzisz ${ }^{2}$ (mentor)

${ }^{1}$ Department of Interventional Radiology and Neuroradiology, Medical University of Lublin, Poland

${ }^{2}$ Department of Paediatric Radiology, Medical University of Lublin, Poland

DOI: https://doi.org/10.26800/LV-142-supp5-43

Introduction:

Crohn's disease is classified as chronic inflammatory bowel disease. The incidence in Europe ranges from 1 to almost 11.4 per 100,000 population per year. Ultrasound examination plays an important role in imaging diagnostics of inflammatory bowel lesions. It allows for assessing response to therapy as well as recognizing possible penetrating complications of the disease, i.e. fistula or abscess.

\section{Materials and Methods:}

36 children were included in the study: 16 boys and 20 girls with an active phase of Crohn's disease. Each patient underwent intestinal ultrasound examination with a high frequency 7-12 Mhz linear probe.

\section{Results:}

In all patients US examination depicted a thickened, hypoechoic ileal wall showing patterns of vascularization. In 8 patients Bauhin' valve edema was visible. In 16 children, inflammatory infiltration of the peri intestinal fat around the affected segment of the intestine was found. In addition, all patients presented mesenteric lymphadenopathy with short-axis diameter of $10-15 \mathrm{~mm}$. 8 patients had penetrating complications of Crohn's disease: 4 small intestine fistulas and 4 abscesses.

\section{Conclusion:}

Given its safety profile and diagnostic efficacy, US examination should be considered as the first-line imaging modality for assessing inflammatory bowel disease in children. The US proved to be a reliable and easily accessible tool in the diagnosis of enteric inflammatory lesions, evaluating CD activity and assessing potential penetrating complications of the disease.

Keywords: Ultrasound, Inflammatory Bowel Disease, pediatric patients 